\title{
Realizing Low-impedance Rendering in Admittance-type Haptic Interfaces Using the Input-to-State Stable Approach
}

\author{
Muhammad Nabeel, Aghil Jafari, Member, IEEE, and Jee-Hwan Ryu, Member, IEEE
}

\begin{abstract}
This paper proposes an approach to enlarge the impedance range of admittance-type haptic interfaces. Admittance-type haptic interfaces have advantages over impedance-type haptic interfaces in the interaction with high impedance virtual environments. However, the performance of admittance-type haptic interfaces is often limited by the lower boundary of the impedance that can be achieved without stability issue. Especially, it is well known that low value of inertia in an admittance model often causes unstable interaction. This paper extends recently proposed input-to-state stable approach [1] to further lower down the achievable impedance in admittance-type haptic interfaces with less conservative constraint compared with the passivity-based approaches. The primary challenge was identifying the nonlinear hysteresis components which are essential for the implementation of the inputto-state stable approach. Through experimental investigation and after separating and merging the admittance model and the position controller, the partial admittance model (from the measured human force to the desired velocity) and the velocity controller (from the velocity tracking error to the controller force) were found having counter-clockwise hysteresis nonlinear behavior. Therefore, it allows implementing the one-port inputto-state stable (ISS) approach for making both components dissipative and ISS. An additional advantage of the proposed ISS approach is the easiness of the implementation. No model information is required, and the network representation is not necessary, unlike the passivity-based approaches. Series of experiments verified the effectiveness of the proposed approach in term of significantly lowering the achievable impedance value compared with what the time-domain passivity approach can render.
\end{abstract}

\section{INTRODUCTION}

Admittance-type haptic interfaces are non-back drivable, operated closed-loop and used to render high virtual impedance such as high inertia and high stiffness. Therefore, the performance of admittance-type haptic interfaces has often been limited by the lower boundary of the impedance that can be achieved without stability issue. Since rendering the lower value of inertia often makes the admittancetype haptic interfaces unstable, minimum achievable inertia without instability issue was the primary design parameter to judge the performance of admittance-type haptic interfaces.

Several studies have been conducted on the limitations of admittance haptic interfaces. Janez [2] investigated the effect of grasp force on the stability of admittance haptic interface. Peer [3] also concluded that the firm operator grasp

Muhammad Nabeel and Jee-Hwan Ryu are with the School of Mechanical Engineering, Korea University of Technology and Education (KoreaTech), Rep. of Korea. Email: \{nabee19172, jhryu\} ekoreatech.ac.kr

Aghil Jafari is with the Faculty of Environment and Technology, University of the West of England, Bristol, United Kingdom. Email: aghil.jafariduwe.ac.uk makes the admittance haptic interfaces unstable. Parthiban [4] proposed a method to evaluate the stable rendering range of the admittance-type haptic displays. Nambi [5] analyzed the effect of velocity, admittance gain, and force on human force control ability. Even though several studies have been done on the stability of admittance rendering, most of the researches were limited to the investigation of the correlation between stability and parameters of the admittance type haptic interface, and only a few of the researches have been conducted to increase the impedance range of admittancetype haptic interfaces.

Recently, time-domain passivity approach (TDPA) was extended to increase the impedance range of the admittancetype haptic interfaces without using any model information [6], and it could successfully lower down the minimum boundary of the impedance about ten times lower than the without case. However, this approach was also limited to further enlarge the impedance range because TDPA was based on passivity constraint, which is known to be conservative.

This paper extends the recently proposed input-to-state stable (ISS) approach [1] to further lower down the minimum boundary of the achievable impedance in admittance-type haptic interfaces. The significant differences of the proposed approach, compared to the previous approaches, is that it is based on the least conservative stability criterion, socalled input-to-state stability criterion, and it does not require any model information for the implementation. Input-tostate stability criterion is known to be less conservative than passivity and absolute stability criterion. Therefore it was able to achieve the lowest value of inertia than the other approaches. One of the primary challenges was investigating the nonlinear hysteresis component of the system which is essential for implementing the developed single-port ISS approach. We divided the admittance model into two parts and independently applied ISS approach after merging second part of the admittance model into the local position/velocity controller of the admittance-type device. Experimental studies validated the effectiveness of the proposed approach. The proposed approach guaranteed ten times lower value of inertia than what time-domain passivity approach can render.

The paper is organized as follows: Section II defines the admittance-type haptic interface and illustrates the limitation. Section III reviews the previously proposed ISS approach for impedance-type haptic interfaces. Section IV introduces the proposed ISS approach to an admittance haptic interface. Section V experimentally evaluates the proposed approach, and Section VI concludes the paper. 


\section{LimitATion OF AdMitTANCE-TyPE HAPtiC INTERFACES}

In an admittance-type haptic interface, an admittance model is designed to generate the desired trajectory $\left(x_{d}\right)$ based on the measured operator force $\left(f_{h}\right)$ from the force sensor (mounted at the tip of the admittance haptic display) as shown in Fig. 1. A local position controller closes the control loop to make the admittance display follow the desired trajectory.

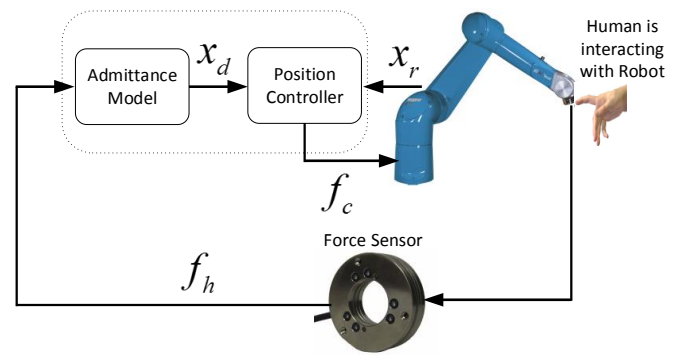

Fig. 1: Conceptual representation of an admittance-type haptic interface

Thanks to the admittance model, the operator can perceive desired virtual impedance or admittance by tuning its parameters. In general, for a linear case, three parameters can be assigned: inertia, damping, and stiffness. To render free space, ideally, all three parameters should be set zero. However, it is technically impossible due to numerical integration, so in general we set all three parameters as small as possible, typically, set the stiffness value as zero while having the inertia and the damping value as small as possible. Once we assume that the bandwidth of the local position controller is fast enough to satisfy the variation of the desired position $x_{d}$, the actual robot position $x_{r}$ can be assumed to be same as the desired trajectory within a certain frequency range. As the result of this assumption, the following impedance relation, between the operator force $\left(f_{h}\right)$ and position $\left(x_{r}=x_{d}\right)$, can be achieved:

$$
f_{h}=m \ddot{x}_{r}+b \dot{x}_{r}+k x_{r},
$$

where $m, b, k, \ddot{x}_{r}, \dot{x}_{r}$ and $x_{r}$ represent inertia, damper, stiffness, actual acceleration, actual velocity and actual position of admittance display, respectively. Following is the simplest first order Euler method to simulate admittance model:

$$
\ddot{x}_{d}=\frac{f_{h}-b \dot{x}_{d}-k x_{d}}{m}, x_{d}=\iint \ddot{x}_{d} d t .
$$

From Eq. (2), we can conclude that we may be able to set the damping and stiffness to zero; however, it is impossible to set the value of inertia to zero due to numerical simulation error. To perceive a free space feeling, value of inertia and damping must be set as small as possible. However, decreasing the value of inertia to certain level makes the admittance-type haptic interfaces unstable [6].

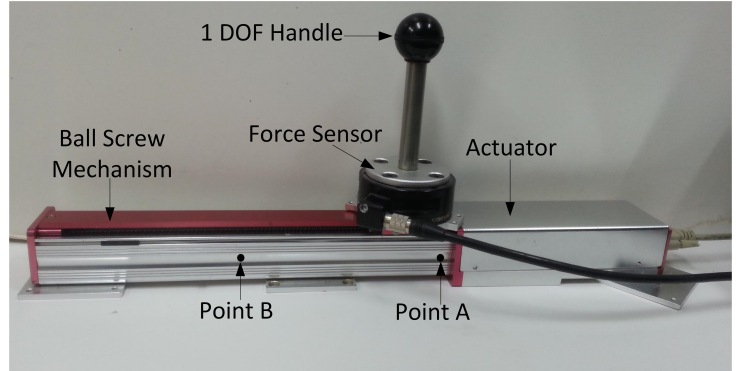

Fig. 2: Experimental setup

To show the limitation of admittance-type haptic interfaces, we have provided an experimental setup as shown in Fig. 2. The experimental setup is a one-DOF admittance-type haptic display geared by lead-crew and driven by a DC motor (FAULHABER 2342CR series). An optical encoder mounted on the motor shaft serves as a position sensor which has a resolution of 512 pulse/revolution. ATI Gamma FT08682, having a sensing range of $32 \mathrm{~N}, 32 \mathrm{~N}$ and $100 \mathrm{~N}$ with resolution of $0.00625 \mathrm{~N}, 0.00625 \mathrm{~N}$ and $0.125 \mathrm{~N}$ in $x, y$ and $z$-axis respectively, is mounted on the gripper as shown in Fig. 2.

Several experiments have been conducted with different values of inertia to demonstrate that the lower values of inertia make the system unstable. For the experiments, we set the stiffness as zero and the damping as 0.01 (Ns/m) (just large enough to compensate the sensor noise). During the experiments, the operator is instructed to push the handle of the haptic device from point $\mathrm{A}$ to $\mathrm{B}$ and then moves it back from point $\mathrm{B}$ to $\mathrm{A}$ twice as shown in Fig.2

As it can be seen in Fig. 3a, for the inertia of $m=0.1 \mathrm{~kg}$, the operator can move the interface as it supposes to move while having some levels of interaction force as shown in Fig. 3b. However, after lowering down the inertia to $0.01 \mathrm{~kg}$ and $0.001 \mathrm{~kg}$, the operator was not able to move the interface as it was instructed because some unexpected interaction force and high-frequency oscillations appeared. Ideally, with the decreased value of inertia, the operator is supposed to feel as if he/she is interacting with the lighter object and applying the smaller force $f_{h}$. However, as shown in Fig. $3 b$, as the value of inertia is decreased to $0.01 \mathrm{~kg}$ and $0.001 \mathrm{~kg}$, operator force $f_{h}$ became even bigger with unintended highfrequency oscillation due to the instability of the system.

\section{ReView of the InPUT-TO-State Stable} APPROACH FOR IMPEDANCE-TYPE HAPITC INTERFACE

In [1], the main concept of the ISS approach was proposed to guarantee the stability of impedance-type haptic interfaces with less conservatism compaed with passivity approaches. Generally, impedance-type haptic interface can be considered as a composition of four components; a human operator, a haptic device, a discrete interface, and a virtual environment as presented in Fig. 4. $f$ and $\dot{x}$ in Fig. 4 represent the force and the velocity of each component. The subscripts $h$ and $d$ mean human operator and haptic device, respectively. 


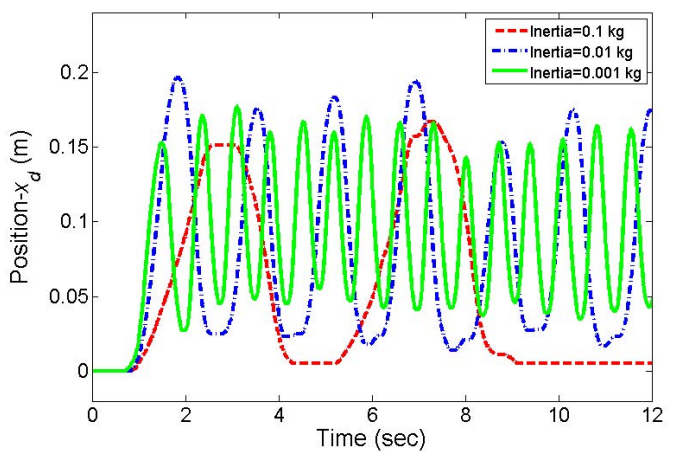

(a) Position response

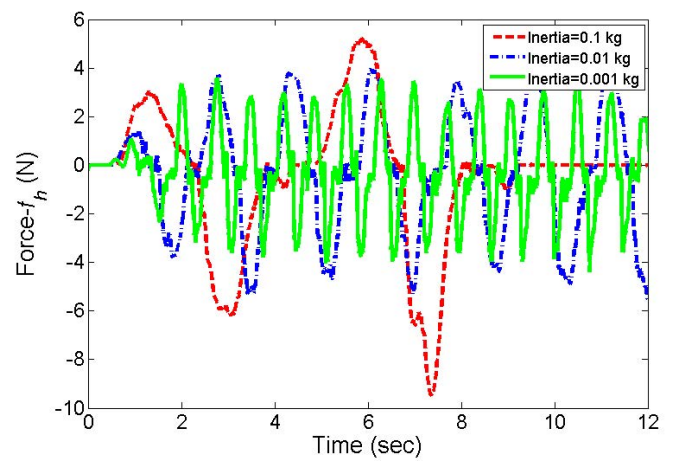

(b) Force response

Fig. 3: Low inertia rendering experimental results; (a) Position response, (b) Force response.

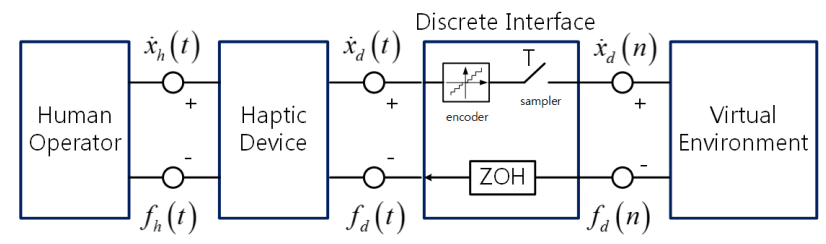

Fig. 4: Overall configuration of impedance-type haptic simulation system.

Based on the concepts presented in [7], if there exists $\alpha<$ $\infty$ such that

$$
\int_{0}^{T} f_{d}(t) \dot{x}_{d}(t) d t \geq-\alpha,
$$

then, we can say that the system is dissipative and ISS. The ISS criterion (the inequality in Eq. (3)) is less conservative than passivity criterion, and passive region can be a subset of ISS region. This comes from the fact that, by holding Eq. (3), as long as $\alpha$ is finite, the system is allowed to generate a finite amount of energy even more than the initial stored energy in the system. Therefore, states are allowed to leave the passive region. However, because Eq. (3) is the sufficient condition for input-to-state stability, the states will be bounded by a function of input, and the system will remain stable.

Fig. 5 shows position $\left(x_{d}\right)$ vs. force $\left(f_{d}\right)$ trajectory of an impedance-type haptic interface after a single interaction, captured in-between the haptic device and the discrete in-

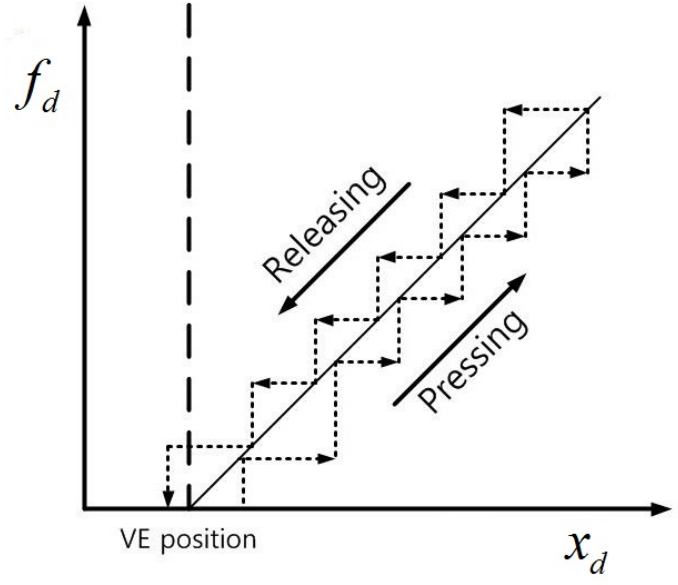

Fig. 5: Position vs. force trajectory of an impedance-type haptic interface in single contact and separation motion. Signals are captured in between haptic device and discrete interface.

terface. It is interesting to see that the pressing path is not the same as the releasing path due to zero-order-hold effect. In this figure, the solid line presents the behavior of the ideal virtual environment, whereas the dashed lines show the actual behavior of discrete virtual environment. The pressing curve starts after the operator establishes a contact with the virtual environment, and the releasing curve ends after the operator is no longer in contact with the virtual environment because of the sensing and computation delay. Therefore, position vs. force behavior of the virtual environment cannot be expressed as a single-value function since pressing path is not the same as the releasing path in Fig. 5. Hence, based on the definition of hysteresis systems [8], the virtual environment can be considered as a system with counterclockwise hysteresis (see Fig. 6), and we can take the benefits of hysteresis properties to design an ISS control architecture for haptic interaction. Based on the concept presented in [8], for systems with hysteresis nonlinearity, time derivative of input-output pairs $\left(\dot{f}_{d}\right.$ and $\left.\dot{x}_{d}\right)$ are sector bounded.

Let $\mu_{\min }$ and $\mu_{\max }$ be the minimum and maximum slopes on the virtual environment characteristic such that $\mu_{\min } \leq f_{d}^{\prime}(x) \leq \mu_{\max }$, and $f_{d}^{\prime}(x)$ is the local slope of the position vs. force graph at position $x_{d}(n)$

$$
f_{d}^{\prime}(x)=\frac{\Delta f_{d}(n)}{\Delta x_{d}(n)}=\frac{f_{d}(n)-f_{d}(n-1)}{x_{d}(n)-x_{d}(n-1)},
$$

where $n$ is the sampling time of the virtual environment. As is typically done under the assumption of a loop transformation, we assume that $\mu_{\min }=0$ [9], then based on [10], sector boundedness of input-output time derivative leads

$$
\dot{f}_{d}(n) \dot{x}_{d}(n)-\frac{1}{\mu_{\max }} \dot{f}_{d}^{2}(n) \geq 0
$$

In [1], for impedance-type haptic displays, an ISS control architecture was proposed which converts a virtual environment into a dissipative virtual environment. Block diagram of the proposed ISS approach [1] is presented in Fig. 7, 


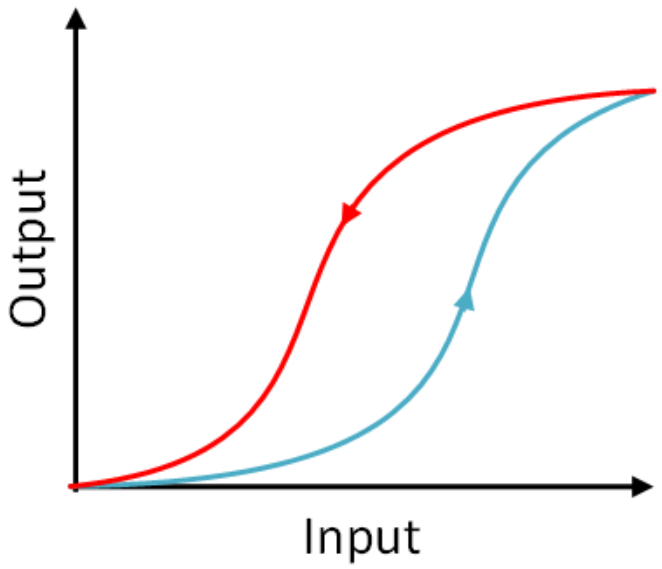

Fig. 6: A graphical illustration of input-output behavior from a counterclockwise hysteresis.

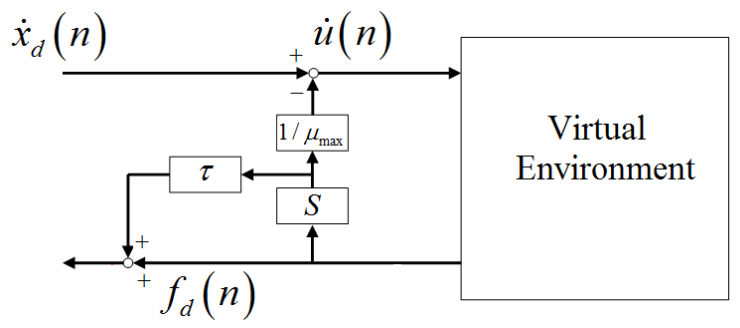

Fig. 7: Control architecture of the ISS approach for impedance-type haptic displays [1]

where $S$ is the Laplace operator, and $\tau$ is a non-negative gain. This control block diagram guarantees the existence of a finite lower bound for the inequality in (3), which is the sufficient condition for the system to be dissipative and ISS. By using hysteresis properties mentioned in [10], the feedback including $\mu_{\max }$ can guarantee the input-to-state stability of the overall system presented in Fig. 7. However, it allows the system to have some bounded oscillations. The amplitude of the bounded oscillations has a direct relation to the magnitude of $\mu_{\max }$, and for very high stiffness virtual environments it may not be pleasant for the operator, even though this amount of oscillations is bounded and not diverging. The feedforward path including $\tau$ is introduced to tackle the bounded oscillations problem, and it is a dissipative feedforward [1]. We must notice that this feedforward path is just introduced to remove the bounded oscillations and it does not contribute to the system stability.

\section{InPUT-TO-STATE StABle APPROACH FOR ADMITTANCE-TYPE HAPTIC INTERFACES}

Fig. 8 shows a general block diagram of an admittancetype haptic interface. In this figure, $f_{h}, f_{c}, x_{r}, x_{d}$ represent the human force measured by a force sensor, the controller force induced by the position controller, the position of the haptic device measured by an encoder, and the desired position computed based on the admittance model, respectively. If each component (human operator, position controller, admittance model and haptic device) of the system is dissipative, we can conclude that overall system is dissipative and ISS. Typically, the human operator, in lowfrequency motion, and the haptic device can be considered as dissipative; however, the admittance model and the local position controller may not be dissipative depending on the gains. For example, high proportional or damping gain of the position controller, and low inertia or damping gain of the admittance model can make each block not dissipative, and it can potentially make the overall system unstable. If we can make both block dissipative and ISS by using the ISS approach, it is possible to make overall system ISS.

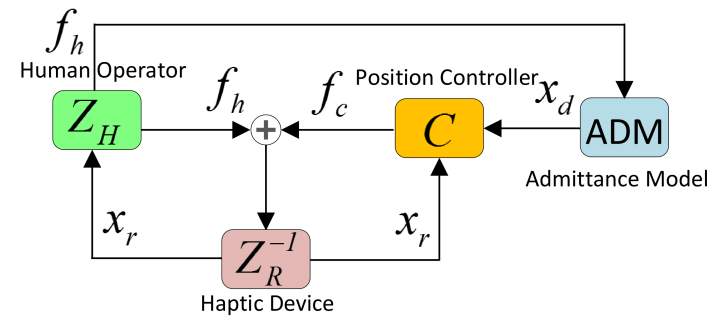

Fig. 8: Block diagram representation of an admittance-type haptic interface.

The first step for the implementation of the ISS approach is to investigate the components with hysteresis nonlinearity among the system components [1]. As we discussed, the position controller and the admittance model are the only two blocks which are potentially non-dissipative.

To find hysteresis behavior, we broke down the admittance model into two parts, the partial admittance model from the measured force $\left(f_{h}\right)$ to the desired velocity $\left(v_{d}\right)$ and the integrator as illustrated in Fig.9, and investigated the nonlinear hysteresis behavior at the partial admittance model. It shows nonlinear hysteresis behavior in the measured force vs. the desired velocity relationship as depicted in Fig. 10. This relationship shows counter-clockwise hysteresis nonlinear behavior. The pressing path was lower than the releasing path, which explains the generated energy and unstable interaction based on the concept presented in [1]. Note that because of the noisy data acquired from the force sensor, the relationship is not clear hysteresis nonlinear curve and it contains noise spikes.

Because we found the partial admittance model to have the nonlinear hysteresis component, now it became possible to implement the feedback path with $\mu_{\max }$ from the previously proposed ISS approach in [1] to the partial admittance model as illustrated in Fig. 9 to make this block dissipative and ISS. $\mu_{\max }$ is the maximum slope of the input-output graph of partial admittance model, decided as follows: 


$$
\begin{aligned}
\mu_{\max } & =\max \left\{\frac{\dot{f}_{h}(n)}{\dot{v}_{d}(n)}\right\}_{i=1, \ldots, n} \\
& =\max \left\{\frac{f_{h}(n)-f_{h}(n-1)}{v_{d}(n)-v_{d}(n-1)}\right\}_{i=1, \ldots, n}
\end{aligned}
$$

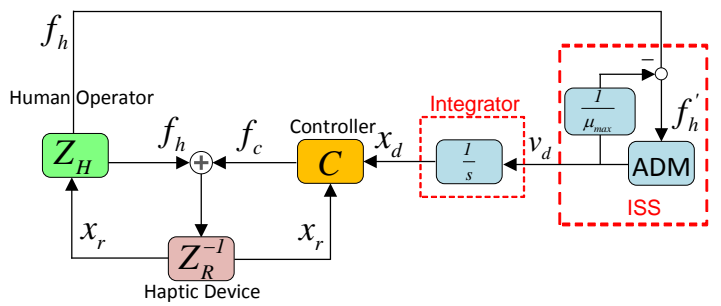

Fig. 9: Block diagram representation of the admittance haptic interface with ISS controller on partial admittance model.

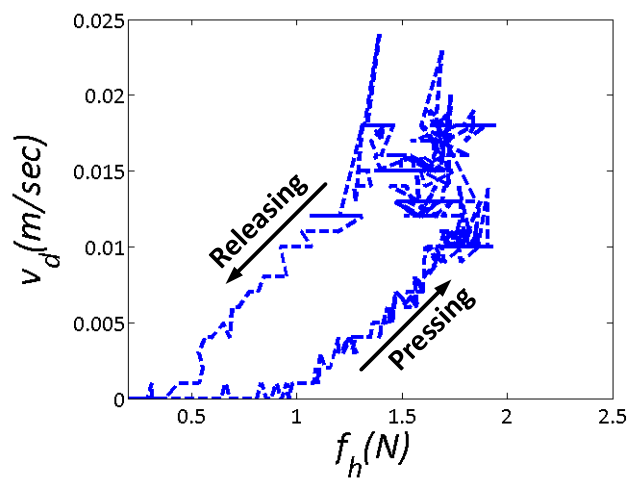

Fig. 10: Input (measure human force, $f_{h}$ ) vs. output (desired velocity, $V_{d}$ ) behavior of the partial admittance model.

Unlike the original ISS approach presented in Fig. 7, the implemented ISS approach in Fig. 9 does not have any time-derivative operator. This comes from the fact that in Fig. 7, time derivation of the input $\left(\dot{x}_{d}\right)$ was revised, but in the implemented ISS approach in Fig. 9, the input itself $\left(f_{h}\right)$ is revised; therefore, there is no need of time-derivative operator.

To make the rest of the system ISS, we combined the integrator and the position controller, and check whether this combined velocity controller shows nonlinear hysteresis behavior. The combined velocity controller (the integrator plus the position controller) shows counter-clockwise hysteresis nonlinear behavior in the input (the velocity tracking error, $\dot{e}_{d}=v_{d}-v_{r}$ ) vs. output (the controller force, $f_{c}$ ) behavior as illustrated in Fig. 11. This hysteresis-like behavior allows implementing another ISS approach to the combined integrator and position controller block as depicted in Fig. 12. In this representation, $\mu_{\max _{c}}$ is the maximum slope of input vs. output graph of the velocity controller, and it is designed to satisfy the ISS condition of the combined integrator and controller block:

$$
\begin{aligned}
& \mu_{\max }=\max \left\{\frac{\dot{f}_{c}(n)}{\ddot{e}_{d}(n)}\right\}_{i=1, \ldots, n} \\
& =\max \left\{\frac{f_{c}(n)-f_{c}(n-1)}{\left(v_{d}(n)-v_{d}(n-1)\right)-\left(v_{r}(n)-v_{r}(n-1)\right)}\right\}_{i=1, \ldots, n}
\end{aligned}
$$

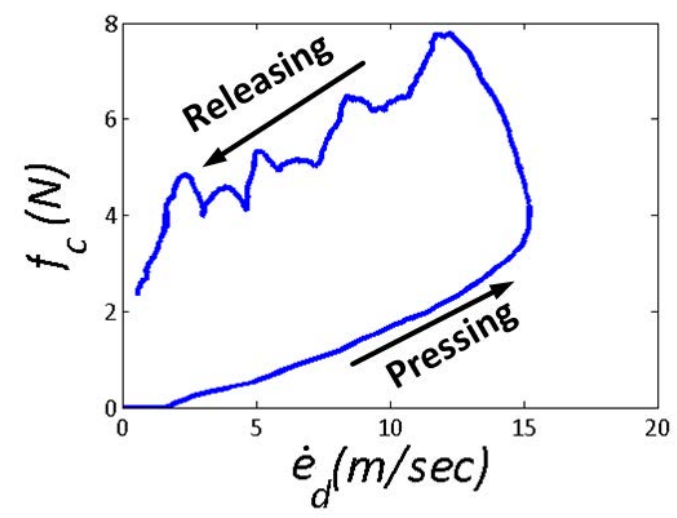

Fig. 11: Input (The velocity tracking error, $\dot{e}_{d}$ ) vs. output (the controller force, $f_{c}$ ) behavior of the integrator and position controller.

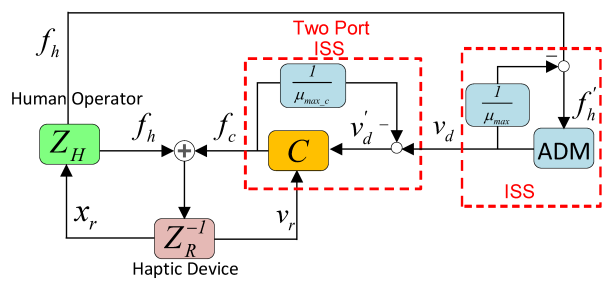

Fig. 12: Block diagram of the dmittance-type haptic interface with the ISS approach on both partial admittance model and the velocity controller.

Fig. 12 shows overall ISS architecture, designed for making the admittance-type haptic interface dissipative and ISS. Two feedback path, with $\mu_{\max }$ for making the partial admittance model ISS and $\mu_{\max _{c}}$ for making the velocity controller ISS, were implemented. For the velocity controller case, there are two options on implementation; revising the tracking error $\left(\dot{e}_{d}\right)$ and revising the desired velocity $\left(v_{d}\right)$. It is easy to show that the controller input for both options would be the same. In this paper, revising the desired velocity $\left(v_{d}\right)$ option is selected because the internal error $\left(\dot{e}_{d}\right)$ may not be available in most of the practical situations. Please note that another feedforward path including a gain $(\tau)$ also was introduced in the original proposed ISS approach [1], and it is not considered in this figure. As it is discussed in Section III, $\tau$ only removes the residual vibration and does not contribute to making the system ISS. 


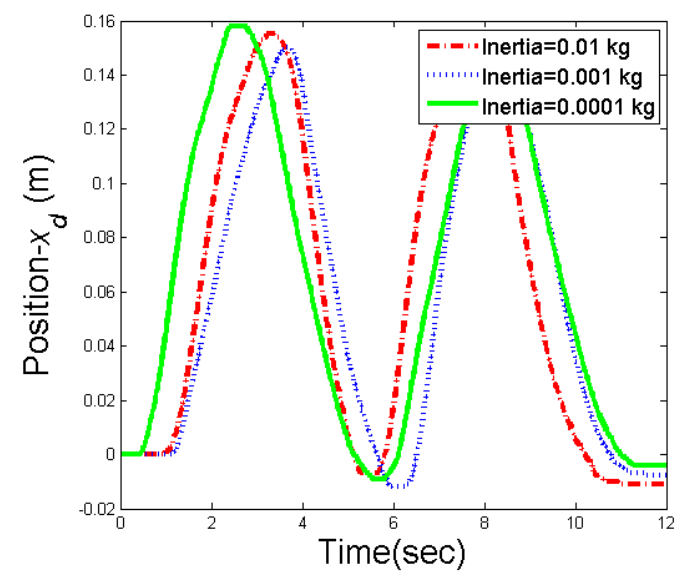

(a) Position response

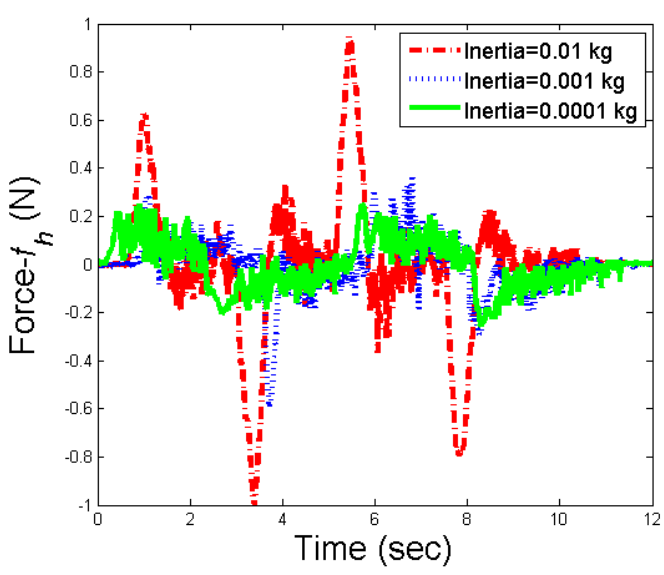

(b) Force response

Fig. 13: Experimental results with the proposed ISS approach; (a) Position responses ( $m=0.0001 \mathrm{~kg}$ ), (b) Force responses.

\section{EXPERIMENTAL EVALUATION}

Fig. 13 illustrates the experimental results with the proposed ISS approach where the experimental setup was the same as in Section II. As it can be seen, the position responses were stable even with the significantly small value of inertia such as $m=0.0001 \mathrm{~kg}$. When it is compared with the results in Fig. 3a, the proposed approach brought down the inertia of the admittance model 1000 times than the conventional case (see Fig. 3a) while guaranteeing the stability at the same time. With the same experimental setup, the TDPA was only able to lower down the minimum value of inertia of the admittance model down to $0.001 \mathrm{~kg}$ [6]. However, thanks to its less conservatism, the proposed ISS approach achieve ten times lower value of inertia.

\section{CONCLUSION}

In this paper, a method to lower down the lower impedance bound of an admittance-type haptic interface was proposed. Through experimental investigation, two components, the partial admittance model (from the measured human force to the desired velocity) and the velocity controller (from the velocity tracking error to the control force) were found having counter-clockwise hysteresis nonlinear behavior. Therefore, it enabled implementing one-port ISS approach for making those components dissipative and ISS. Series of experiments demonstrated the effectiveness of the proposed approach. The proposed ISS approach was able to lower down the impedance value ten times for both inertia and damping coefficient compared with what the TDPA can render. The additional advantage of the proposed ISS approach is that no model information is required and the network representation is not necessary, unlike the TDPA.

\section{ACKNOWLEDGMENT}

This research supported by the project (Development of core teleoperation technologies for maintaining and repairing tasks in nuclear power plants) funded by the Ministry of Trade, Industry \& Energy of S. Korea.

\section{REFERENCES}

[1] A. Jafari and J. H. Ryu, "Input-to-state stable approach to release the conservatism of passivity-based stable haptic interaction," in 2015 IEEE International Conference on Robotics and Automation (ICRA), pp. 285-290, May 2015.

[2] J. Podobnik and M. Munih, "Haptic interaction stability with respect to grasp force," IEEE Transactions on Systems, Man, and Cybernetics, Part C: Applications and Reviews, vol. 37, pp. 1214-1222, Nov 2007.

[3] A. Peer and M. Buss, "A new admittance-type haptic interface for bimanual manipulations," IEEE/ASME Transactions on Mechatronics, vol. 13, pp. 416-428, Aug 2008.

[4] C. Parthiban and M. Zinn, "A simplified approach to admittance-type haptic device impedance evaluation," in IEEE Haptics Symposium (HAPTICS), pp. 587-590, Feb 2014.

[5] M. Nambi, W. R. Provancher, and J. J. Abbott, "On the ability of humans to apply controlled forces to admittance-type devices," Advanced Robotics, vol. 25, no. 5, pp. 629-650, 2011.

[6] M. Nabeel, J. Lee, U. Mehmood, A. Jafari, and J.-H. Ryu, "Feedback systems: Input-output properties," in IEEE/RSJ International Conference on Intelligent Robots and Systems, Hamburg, Germany, 2015.

[7] E.D.Sontag, "Nonlinear and optimal control theory: Input to state stability," in Springer, pages 163-220, 2006.

[8] T. E. Pare, "Passivity-based analysis and control of nonlinear systems," in Published doctoral dissertation, Stanford University, California, U.S, 2000.

[9] M. Vidyasagar, "Nonlinear systems analysis," in 2nd Ed. Academic Press, New York, NY, 1993.

[10] T. E.Pare and P. P.How, "Robust stability and performance analysis of systems with hysteresis nonlinearities," in American Control Conference (ACC), pages 1904-1908, 1998. 